\title{
EVOLUCIÓN DE LAS HABILIDADES LABORALES EN LA INDUSTRIA 4.0 Y SU IMPACTO FINANCIERO
}

\section{EVOLUTION OF LABOR SKILLS IN INDUSTRY 4.0 AND ITS FINANCIAL IMPACT}

\author{
Paris Anaid Castellanos Rivero ${ }^{1}$ \\ María del Pilar Escott Mota ${ }^{2}$
}

Cómo citar este artículo: Castellanos Rivero, P. A., \& Escott Mota, M. del P. (2020). Evolución de las habilidades laborales en la industria 4.0 y su impacto financiero. Revista Innova ITFIP, 6(1), 106-119. Recuperado a partir de http://revistainnovaitfip.com/index.php/innovajournal/article/view/82

\section{Recibido: Enero 2020 Aprobado: Junio 2020}

\section{Resumen}

La industria 4.0 se está preparando para transformar el trabajo a un ritmo acelerado a través de tecnologías que crecen exponencialmente; como la inteligencia artificial, la robótica avanzada, la automatización cognitiva, el análisis avanzado y el Internet de las cosas. Es probable que la tecnología cree más empleos de los que destruye, como lo ha hecho históricamente, de tal forma que quienes van a perder sus trabajos en esta transición podrían ser los menos preparados para aprovechar las nuevas oportunidades que las nuevas tecnologías ofrecen. Por consiguiente, la presente investigación tiene como objetivo explorar e identificar las habilidades futuras de capacitación para la industria 4.0 y el impacto financiero que les ha generado. La exploración de literatura permite concluir que las competencias de hoy no se ajustarán a los trabajos del mañana y las nuevas competencias adquiridas pueden quedar desfasadas rápidamente. Las empresas, el trabajo y la economía se están viendo impactados por la automatización y la inteligencia artificial. La tecnología esta inmiscuida con todas las funciones de las organizaciones y cada día será más importante. Por esta razón, después del reciente acontecimiento del COVID -19, los gobiernos, las empresas y la sociedad deben prepararse mejor tecnológicamente a medida que se tomen acciones para evitar la disrupción de las operaciones y continuar laborando en tiempos en donde existe un distanciamiento social.

\footnotetext{
${ }^{1}$ Docente de la Universidad Autónoma de Querétaro Facultad de Contaduría y Administración. México. Correo: paris.anaid.castellanos@uaq.mx.

${ }^{2}$ Docente de la Universidad Autónoma de Querétaro Facultad de Contaduría y Administración. México. Correo: maria.delpilar.escott@uaq.mx.
} 
Palabras clave: Habilidades; automatización; internet de las cosas y disrupción.

\begin{abstract}
Industry 4.0 is preparing to transform work at an accelerated rate through technologies that grow exponentially; such as artificial intelligence, advanced robotics, cognitive automation, advanced analytics, and the Internet of Things. Technology is likely to create more jobs than it destroys, as it has historically done, so that those who will lose their jobs in this transition may be the least prepared to take advantage of the new opportunities that new technologies offer. Therefore, this research aims to explore and identify future training skills for Industry 4.0 and the financial impact it has generated. Exploring the literature allows us to conclude that the skills of today will not fit the jobs of tomorrow and the new skills acquired may quickly become obsolete. Business, work and the economy are being impacted by automation and artificial intelligence. Technology is immersed in all the functions of organizations and will become more important every day. For this reason, after the recent event of COVID -19, governments, companies and society must prepare better technologically as actions are taken to avoid disruption of operations and continue working in times of social distancing.
\end{abstract}

Keywords: Skills; automation; internet of things \& disruption.

\section{Introducción}

El uso de herramientas virtuales se ha convertido en un elemento clave para el desarrollo económico de los países y específicamente del sector empresarial, su implementación y uso incrementa la competitividad $\mathrm{y}$ tiene efectos positivos en el crecimiento económico, integración social y sostenibilidad ambiental; además mejora las acciones de RSE (Responsabilidad Social Empresarial). (Ceballos, Mejía \& Arango 2019 p. 65).

En ese sentido, la creciente penetración de nuevas tecnologías emergentes en los entornos de producción $\mathrm{y}$ distribución de fabricación han creado una demanda de profesionales que se destaca en este panorama de tecnologías y fabricación en evolución distinguiéndose por su capacidad para comprender la nueva tecnología (Weller, Dollar, Manolian, Mock \& Hussain., 2019). Las economías crearan millones de puestos de trabajo a medida que se adopten prácticas tecnológicas sostenibles y limpias; en cambio, otros puestos de trabajo desaparecerán cuando los países vayan reduciendo progresivamente sus industrias basadas en el carbón y en el uso intensivo de los recursos (Ryder y Sarah, 2019). Como señala Bughin, Hazan, Lund, Dahlström, Wiesinge \& Subramaniam (2018) a casi una de cada tres empresas les preocupa que la falta de las habilidades requeridas para la adopción de la automatización afectará su desempeño financiero futuro. $\mathrm{La}$ automatización acelerará el cambio en las habilidades requeridas de la fuerza laboral que se ha visto en los últimos 15 años (Bughin et al., 2018). Los ingresos crecientes, las inversiones en infraestructura, la energía y otros catalizadores podrían crear potencialmente millones de nuevos trabajos si bien el desplazamiento de 
mano de obra de la automatización ha sido visible durante muchos años, es más difícil imaginar todos los nuevos empleos que se crearán, muchos de estos nuevos empleos se crean indirectamente y se extienden a través de diferentes sectores y geografías (Manyika, Lund, \& Chui. 2017).

Con base a PwC (2017) a nivel mundial la Inteligencia Artificial (IA) provocará un incremento adicional del PIB mundial en 2030 de 15,7 billones de dólares adicionales además el 42\% generarán como consecuencia del incremento de la productividad y 9,1 billones por los efectos en el consumo. Los resultados sobre la tecnología y el trabajo sugieren una perspectiva del panorama actualmente y hacia dónde se dirige en un futuro cercano. De esta manera se evidencia aspectos y áreas en las que las organizaciones, los gobiernos, las comunidades y los trabajadores deberán trabajar arduamente para adaptarse a la industria 4.0 (PwC, 2018). El trabajo que aquí se presenta tiene como objetivo explorar la evolución de las habilidades laborales requeridas para poder enfrentar los cambios tecnológicos y así tener mayor productividad y por consecuencia un retorno de la inversión más rápida. También se explora el COVID-19 desde una perspectiva positiva, visto como una oportunidad de disrupción tecnológica.

\section{Marco teórico}

\subsection{Concepto de Industria 4.0}

El concepto de industria 4.0 según Mexican Ministry of Economy (2016) lo describe como el fenómeno que existe entre diversos factores gracias a un impacto trasversal de tecnologías de la información, internet de las cosas (IoT, por sus siglas en inglés) en diferentes sectores industriales. Como menciona Morrar, Arman \& Mousa (2017) la Industria 4.0 (I 4.0) está relacionada con lo que se llama la fábrica inteligente ya que es una copia virtual del mundo físico y la toma de decisiones descentralizada, además, sistemas físicos pueden cooperar y comunicarse entre sí y con las personas en tiempo real. OCDE (2018) reporta que la digitalización es parte fundamental de la I 4.0 que está impulsando el cambio estructural, lo que lleva a la creación de nuevos sectores y nuevas fuentes de crecimiento para industrias tradicionales, pero también la desaparición de otras. Este cambio tecnológico estructural ofrece oportunidades para que los países profundicen $\mathrm{y}$ participen de manera más activa en las cadenas de valor globales e impulsen la inclusión de las sociedades, pero también expone a los países a riesgos que deben ser gestionados (Mexican Ministry of Economy, 2016). Asimismo, surgirán nuevos empleos a medida que se desarrolle la revolución digital (OCDE, 2018). PwC (2016) por su parte menciona que la industria 3.0 se centró en la automatización de máquinas y procesos individuales, mientras que la Industria 4.0 se centra en la digitalización de extremo a extremo de todos los activos físicos y la integración 
en ecosistemas digitales con socios de la cadena de valor donde la generación, el análisis y la comunicación de datos respaldan a la perfección las ganancias prometidas por industria 4.0, que conecta una amplia gama de nuevas tecnologías para crear valor $\mathrm{PwC}$ (2016). En concordancia con lo anterior, Martínez (2019), hace una actualización de las concepciones de comunicación, ciencia, tecnología y públicos que están vigentes en la divulgación de la ciencia en México, gracias a las consideraciones que aporta la teoría del actor-red para mejorar los procesos divulgativos a través de las Tecnologías de la Información y la Comunicación, con las que se actualizan los modelos divulgativos de forma que se sintonicen con los escenarios de la cuarta revolución industrial", (p.78)

El segundo aspecto es que la industria 4.0: “[...] ]ofrecerá una gran flexibilidad y robustez, junto con los más altos estándares de calidad en ingeniería, gestión, fabricación, operaciones $\mathrm{y}$ procesos logísticos. Detonará cadenas de valor dinámicas, optimizadas en tiempo real y con una organización automatizada, que considerará varias variables como costos, disponibilidad, y uso de recursos y demanda del mercado este cambio de paradigma representa una ventana de oportunidad para México con un potencial considerable, ya que permitiría la convergencia de dos sectores estratégicos claramente competitivos en el país: Fabricación y Tecnologías de la Información y la Comunicación" [...] Mexican Ministry of Economy (2016, p.10).

\subsection{Las Habilidades del futuro}

Existe consenso sobre el hecho de que generar y adoptar nuevas tecnologías, incluidas las digitales, requiere que los trabajadores estén dotados de ciertas habilidades que les permitan hacer frente y prosperar en la transformación digital (OCDE 2018). Además, como la transformación digital afectará en última instancia a todas las industrias, incluidas aquellas que actualmente son menos intensivas digitalmente, es importante que la política pública comprenda en qué habilidades invertir y cómo ayudar a adaptar las habilidades de los trabajadores a las oportunidades y desafíos impuestos por la transformación digital (OCDE 2018). Como plantea Bughin et al. (2018) para adoptar y comprender la naturaleza y la magnitud del próximo cambio de habilidades, se adoptó un enfoque orientado a los negocios y se incluyeron tanto habilidades intrínsecas - por ejemplo, habilidades motoras gruesas y fuerza, creatividad y empatía - como habilidades aprendidas específicas informática avanzada y programación, análisis avanzado de datos y diseño de tecnología -. Esto permite construir una visión integral de la naturaleza cambiante de las habilidades de la fuerza laboral y proporcionar un nivel de detalle suficiente para motivar acciones e intervenciones concretas (Bughin et al., 2018). Por tanto, los trabajadores exhiben diversas habilidades para laborar de manera ágil y las organizaciones tiene sólidas capacidades en análisis de datos, interacción humano-máquina y toma de decisiones con soporte tecnológico (Geissbauer, Lübben, Schrauf, 
Pillsbury, 2018) Como plantea Bughinet al, (2018) se determinaron 25 habilidades en cinco categorías amplias: físicas y manuales, cognitivas básicas, cognitivas superiores, sociales y emocionales, y tecnológicas. Dentro de cada categoría hay habilidades más específicas, el aprendizaje continuo, la enseñanza y capacitación, posteriormente se separaron las habilidades tecnológicas de las habilidades cognitivas superiores, aunque algunas de las primeras requieren capacidades cognitivas superiores (Bughin et al., 2018). En la siguiente tabla 1 se detallan estas habilidades:

Tabla 1. Habilidades laborales futuras

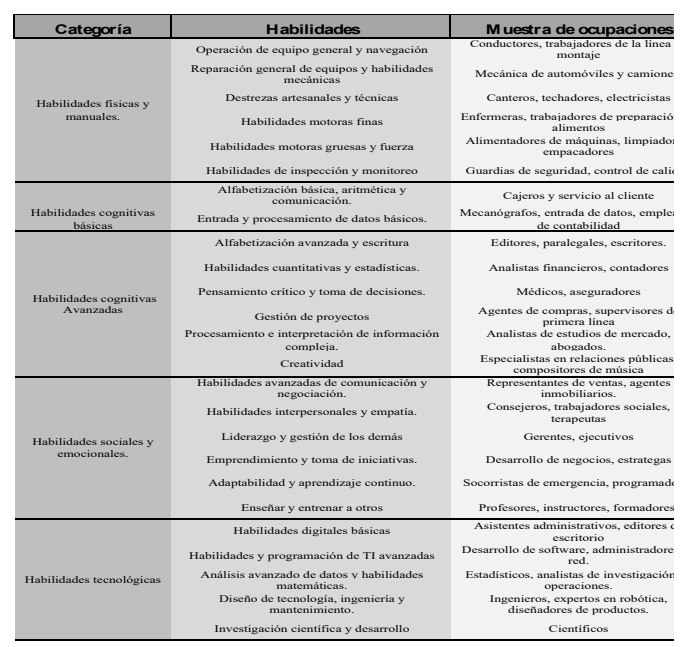

Fuente: Elaboración propia a partir el modelo de habilidades laborales de McKinsey Global Instituto.

Como se puede apreciar en la tabla 1 el mayor cambio tendrá lugar en las habilidades tecnológicas, tanto en habilidades avanzadas como programación y análisis avanzado de datos y diseño tecnológico (Bughin et al., 2018). Las habilidades de manera general verán un aumento significativo en la demanda, incluyendo varios tipos de habilidades sociales y emocionales (Bughin et al., 2018). Se producirá un cambio desde las habilidades cognitivas básicas a las superiores (Bughin et al., 2018). La demanda de habilidades físicas y manuales como un conjunto de habilidades predominantes continuará disminuyendo, aunque estas habilidades seguirán siendo un componente importante del lugar de trabajo del futuro (Bughin et al., 2018)

Hagel, Seely \& Wooll (2019) mencionan que puede parecer que el entorno actual, con el avance tecnológico que impulsa necesidades de nuevos dominios tecnológicos que exigen habilidades más variadas exigen a las empresas redoblar esfuerzos para capacitar. En este sentido, Deloitte (2018) da a conocer que muchas organizaciones líderes implementaron un área de recursos humanos de alto impacto a través de movimientos hacia comunidades de experiencia, el futuro exige un trabajo de reinvención, avanzando más allá la estructura organizacional ya que está en constante cambio y avanzando hacia las mejoras para realizar el trabajo y a si cumplir con la constante disrupción de la empresa. En este nuevo mundo, el ritmo del cambio exige un fuerte ecosistema de socios para construir y permitir capacidades internas con velocidad, mientras crea nuevos canales para talento e innovación (Deloitte, 2018) 


\subsection{Covid 19 y su impacto en el sector tecnológico}

Las compañías industriales esperaban que el año 2020 ejerciera presión económica por las disputas comerciales en curso, las secuelas del Brexit, los desafíos de la industria automotriz y la desaceleración de la demanda en China, pero nadie anticipó que la pandemia de COVID-19 arrojaría a la economía global y sus propias operaciones a una crisis sin precedentes (Fahrni, Jansen, Michael, Kasah Körber \& Mohr, 2020). A medida que el coronavirus continúa propagándose, los gobiernos, las autoridades sanitarias y los líderes empresariales se centran en preservar vidas y contener la pandemia (Fahrni, Jansen, Michael, Kasah Körber \& Mohr, 2020). Paralelamente, quieren disminuir el número de víctimas humanas protegiendo los medios de vida y a la economía de millones de trabajadores que ahora están desempleados, desempleados $o$ en peligro de perder sus empleos (Fahrni, Jansen, Michael, Kasah Körber \& Mohr, 2020).

Mencionando el reporte de Deloitte (2020) sobre los efectos alrededor del mundo del COVID-19 en el sector tecnológico desde los impactos de suministro de materias primas, la interrupción de cadena de valor de la electrónica, el alta inflacionaria de los productos, existe una perspectiva positiva ya que la disrupción ha causado la aceleración del trabajo remoto, además de un enfoque rápido sobre eliminar el riesgo de cadena de valor de extremo a extremo algo semejante ocurre con la contaminación ya que hay una potencial reducción de emisiones de carbono y retomar un nuevo enfoque en prácticas sustentables y tecnologías limpias (Deloitte, 2020). En cuanto a la preocupación por la recesión global menciona PwC (2020b) también se incrementa ligeramente - del $70 \%$ al $73 \%$-, mientras que se reduce la relacionada con el consumo cae de forma significativa - del $52 \%$ al $38 \%-$ .Ante la crisis de COVID-19, los líderes industriales tienen un imperativo comercial: mantener sus operaciones. El internet de las cosas (IoT)y el implementar en un modo plug-and-play - enchufar, conectar y usar -, puede ser instrumental para garantizar la continuidad del negocio y minimizar el daño económico al garantizar la seguridad de los empleados, mejorar la liquidez y reducir los costos a corto plazo. Fahrni et al. (2020) afirma que: "Una empresa escalonó rápidamente los descansos y reorganizó los cambios en función de los conocimientos de IoT, lo que le permite continuar las operaciones y reducir drásticamente el contacto de los empleados" (p.4).

Por estas razones como menciona Deloitte (2020) el software de seguridad advertirá beneficios con la creciente fuerza de trabajo remota, su gasto en software de seguridad aumentara la carrera de las organizaciones publicitarias para asegurar los datos basados en la nube, gestión de registros y red privada virtual (RPV), lo que significa que las compañías con tecnología para trabajo remoto están listas para incrementar las 
demanda en sus negocios he incrementar sus capacidades en trabajo remoto. Un aspecto clave son los servicios de Tecnologías de la información ya que existen pronósticos de la continua demanda de servicios de infraestructura en la nube y aumentos penitenciales en gastos de software especializado además de incrementar la demanda de equipos de comunicación y servicios de telecomunicaciones impulsando que los empleados trabajar desde casa y permita que los niños tomar cursos en línea (Deloitte, 2020).

Fahrni et al. (2020) puntualiza un elemento central y es que entre más digitalizados son los procesos de una empresa, más sencillo es colaborar de forma remota, las herramientas como el internet de las cosas y la interconexión permitirán la continuación de las operaciones con menos empleados en el sitio, ya que facilitan el trabajo remoto en funciones directas e indirectas

\subsection{Impacto financiero}

Según Calderón (2018), "la inclusión financiera como estrategia del sistema financiero ha sido muy acertada $y$ conveniente, ha evolucionado en los últimos años de manera acelerada generando progreso". (p. 48) En ese sentido, las inversiones en la Industria 4.0 ya son significativas, la investigación realizada por PwC (2019) sugiere que las compañías globales de productos industriales invertirán US \$ 907 mil millones por año hasta 2020. El foco principal de esta inversión será en tecnologías digitales como sensores o dispositivos de conectividad, así como en software y aplicaciones. Esto apoya lo que menciona Bughin et al. (2018) respecto a que los riesgos son altos tanto para las empresas como para los trabajadores, cuyos salarios podrían estancarse o incluso disminuir, si no pueden mejorar sus habilidades para cumplir con los requisitos para la Industria 4.0. Al igual que en los Países Bajos, el reentrenamiento y la mejora de las habilidades podrían dar un impulso sustancial a la productividad, así como a una fuerza laboral y asegurar que los trabajadores puedan emplearse más fácilmente. En general, esto podría generar un retorno de la inversión en capacitación de habilidades que se estima en un 30 por ciento después de impuestos (Bughin et al., 2018).

En relación con lo que reporta $\mathrm{PwC}$ (2016) los promedios de las compañías esperan reducir los costos operativos en un 3.6\% anual, al tiempo que aumentan la eficiencia en un $4.1 \%$ anual. Se esperan altos niveles de reducción de costos en todos los sectores industriales - ver figura 1 -, algunos de estos ahorros de costos se pueden lograr mediante la implementación de iniciativas de fabricación inteligente (PwC, 2016). La planificación horizontal y en tiempo real basada en el sistema y la colaboración horizontal ahora es posible utilizando plataformas de planificación basadas en la nube (PwC, 2016). Además, la implementación de dispositivos de seguimiento y localización en los productos conducirá a un mejor rendimiento del inventario y a un menor costo logístico aseguran $\mathrm{PwC}$ (2016). 
Figura 1: Las empresas en todos los sectores industriales esperan reducciones significativas de costos

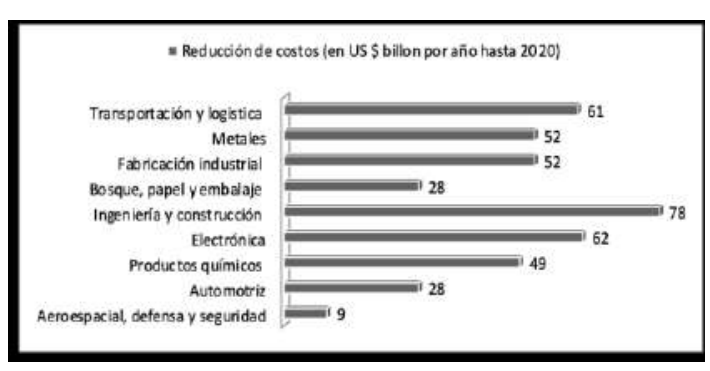

Fuente: Elaboración propia basada en a PwC (2016, p. 13)

\subsubsection{Retorno de la inversión.}

Según Canto y Mejía (2007) la rentabilidad del activo o ROI (del inglés Retornó on Investment) es un indicador muy utilizado en la evaluación de empresas. $\mathrm{La}$ financiación pasa a segundo plano y no se considera el costo del dinero ni el nivel de riesgo con la que opera la empresa. En este sentido Moreno y Rivas. (2002) Mencionan que inversionistas patrimoniales al momento de tomar la decisión de invertir necesitan saber que tan eficientes son las compañías en el uso de los recursos, para evaluar la eficiencia de los recursos financieros, se tendría que aplicar el método en el cual se trata de calcular la tasa de rendimiento sobre estos recursos y esta tasa de rendimiento se le llama el rendimiento de la inversión, o (ROI).

El cálculo del rendimiento de inversión matemáticamente es un concepto simple y nos indica que: el rendimiento anual o la utilidad generado por la inversión es un porcentaje de la suma promedio invertida a lo largo de un año
(Moreno y Rivas, 2002). La idea básica se ilustra mediante la fórmula: Rendimiento de la inversión (ROI) = Rendimiento / Valor promedio invertido (Moreno y Rivas, 2002).

Un estudio realizado por PwC (2016) menciona que la mayoría de las compañías creen que verán un retorno de la inversión (ROI) dentro de dos años o menos - ver Figura 3 - para sus proyectos de Industria 4.0, además poco más de un tercio de las empresas anticipan un plazo más largo de tres a cinco años, pero muy pocas piensan que las inversiones de la Industria 4.0 tardarán más de cinco años en retornarse.

Figura 2 ROI esperado en la inversión de la Industria 4.0

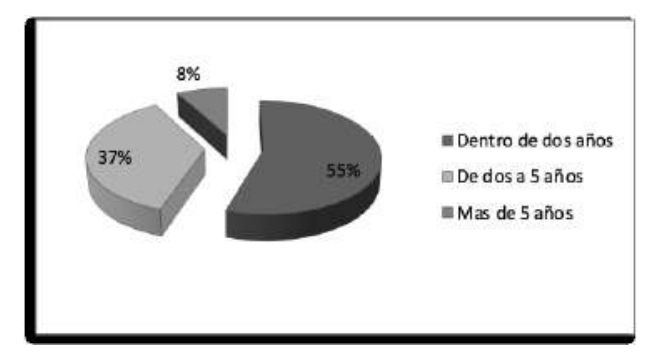

Elaboración propia basada (PwC, 2016 p. 25)

En la figura se puede apreciar que, un poco más de un tercio de las compañías aún esperan mantener su inversión futura relativamente baja (PwC, 2016). Algunas de estas compañías pueden estar esperando la tecnología "perfecta", el mayor desafío que enfrentan las empresas no es comprar la tecnología adecuada, sino transformar su organización y cultura, esto requiere programas de cambio a largo plazo (PwC, 2016) 


\section{Materiales y Métodos.}

Esta investigación se realizó a partir del método de investigación documental propuesta por Hoyos (2000), el cual versa en que a través de distintas fuentes de información como son plataformas electrónicas de libros, revistas especializadas, reportes análisis de indicadores, se lleva cabo análisis crítico de la literatura a partir de cuatro fases planteadas por Marquina, Alvarez, Guevara y Guevara (2013): búsqueda de la literatura, exploración de la literatura, desarrollo del argumento y crítica de la literatura.

Para llevar a cabo el análisis y atender el objetivo de identificar las habilidades futuras de capacitación para la industria $4.0 \mathrm{y}$ el impacto financiero que les ha generado, se realizó búsqueda profunda de expertos en el tema Industria 4.0 como Weller, Dollar, Manolian, Mock \& Hussain, (2019), Weier, Dinesh, \& Venkataraju, (2019), Fahrni, Jansen, Michael, Kasah, Körber \& Mohr (2020). Además, Granados, Varghese, Baranova, Dechalendar \& Wallenstein, (2019), Moueddene, Coppola, Wauters, Ivanova, Paquette \& Ansaloni (2019). Geissbauer, Lübben, Schrauf, Pillsbury (2018), Lund, Manyika, Hilton, Dua, Hancock, B., Rutherford \& Macon (2019), Morrar, Arman \& Mousa, (2017). Bughin, Hazan, Dahlström, Wiesinger \& Subramaniam, (2018), Ryder, Sarah, (2019). Para la obtención de datos se considera Mexican Ministry of Economy, la Organización para la Cooperación y el Desarrollo Económicos (OCDE), Price Waterhouse Coopers, Delloite, además de los reportes actuales de COVID- 19.
Conjuntamente de informes de las habilidades del futuro en la manufactura, estudios sobre el futro de trabajo en América igualmente del análisis de la construcción de las empresas digitales, el futuro de las empresas y las demandas de los recursos humanos del futuro. Finalmente, se estudian las formas de como impactan los cambios tecnológicos y la innovación sobre las competencias ocupacionales y se analiza de qué manera tal impacto puede y debe ser regulado por los actores sociales, en tanto ellos son partícipes activos de la sociedad del conocimiento a través de Illanes, Mourshed, Rutherford y Tyreman (2018).

\section{Resultados y discusión}

En definitiva, los trabajadores que tienen una perspectiva positiva de como impactara la automatización en el lugar de trabajo, tienden a responder positivamente sobre la necesidad de reeducarse y volver a capacitarse para responder a las demandas de habilidades de la industria 4.0, además se espera que los gobiernos tengan un papel a través de la protección y de nuevos beneficios sociales como responsables de formulación de políticas y de diseñar nuevos programas para atenuar los efectos del cambio tecnológico (Granados, Varghese, Baranova, Dechalendar, Wallenstein, 2019).

Referente con lo que menciona Pwc (2018) respecto a la pérdida de empleo debido a los avances tecnológicos 
existe la relación de que muchas actividades se están automatizando además de crearse nuevas funciones y actividades, las empresas tienen la responsabilidad de proteger a su personal y darles las herramientas para la recapacitación y la adaptabilidad. En este sentido es inevitable que algunos empleos desaparezcan de ahí surge la necesidad que las habilidades tecnológicas respondan a los nuevos requerimientos que la misma tecnología genera ya que la cantidad y el valor de empleo se transformara y es un imperativo el acoplamiento ( $\mathrm{PwC}$, 2018). Esto apoya lo que indica PwC (2019):

[...]"Por su parte los gobiernos deberán desarrollar un acercamiento responsable y políticas que regulen el impacto de la tecnología y la automatización, incluyendo las cuestiones éticas de la IA". "También deberán afrontar el tema del desempleo, lo cual puede implicar poner a prueba redes de seguridad social e identificar nuevas fuentes de ingresos para los ciudadanos. Los países menos desarrollados deberán esforzarse aún más para procurar alcanzar al resto, e incluso considerar medidas radicales"[...] (p.15).

En otros retos vinculados a la fuerza laboral, la gran mayoría de las empresas pareciera que tienen de alguna $u$ otra forma resuelto el reto del trabajo a distancia durante esta crisis, con una importante reducción del 55\% al $24 \%$ en la preocupación de contar con las herramientas para trabajar a distancia, sin embargo, para los directivos que tuvieron que desarrollar de forma acelerada políticas de trabajo remoto, así como adoptar nuevas herramientas y software, ahora es el momento de garantizar que las personas sean eficientes y cuenten con la seguridad necesaria para trabajar desde sus casas (PwC, 2020b).

\section{Conclusiones y Recomendaciones}

Según el reporte de la OCDE (2018) sugiere que las habilidades y las tecnologías digitales son complementos en el proceso de producción. Por lo tanto, a la luz de la transformación digital progresiva de la producción, es importante continuar invirtiendo en las habilidades necesarias para hacer frente a las oportunidades y los desafíos de la digitalización. La política debe volver a considerar cómo equipar mejor a las personas con la amplia gama de habilidades necesarias para hacer frente y prosperar en la transformación digital. A medida que evolucionen las tecnologías digitales, también lo harán las habilidades necesarias para trabajar y vivir en este nuevo paradigma tecnológico. Esto es cierto no solo para las habilidades cognitivas, especialmente las cuantitativas, sino también para las habilidades socioemocionales (OCDE, 2018).

En este sentido, los gobiernos deben enfocarse centrarse de forma positiva sobre la industria 4.0 recalcando las oportunidades y cómo responder a ellas. Los gobiernos deben tomar medidas para garantizar que todos 
conozcan y estén preparados ayudando a los trabajadores que corren riesgo de verse afectados con medidas de política personalizadas (Moueddene, Coppola, Wauters, Ivanova, Paquette. \& Ansaloni, 2019). Una forma para que los líderes políticos ganen confianza es adoptar el cambio digital mediante la adopción de tecnologías para mejorar la prestación de servicios públicos y fomentar la innovación (Moueddene et al., 2019). Los encargados de formular políticas deben involucrar a otros actores clave, como universidades, empresas y centros profesionales, para facilitar la entrega de esquemas de capacitación y crear conciencia sobre la importancia del aprendizaje continuo (Moueddene et al., 2019).

Desde la perspectiva de los líderes industriales, están digitalizando las funciones dentro de los procesos internos de las operaciones, además se están mejorando su cartera de productos funcionales digitales e introduciendo servicios innovadores basados en datos. Las más de 2,000 compañías que se encuestaron esperan aumentar drásticamente su nivel general de digitalización. Si bien solo el $33 \%$ califica a su empresa como avanzada hoy en día, ese número salta a más del 70\% mirando hacia 2020 PwC (2016).

A modo de conclusión, como mencionan Lund, Manyika, Hilton, Dua, Hancock, Rutherford \& Macon (2019), la adopción de tecnologías de inteligencia artificial va a significar, probablemente, la desaparición de aquellos puestos de trabajo que puedan ser redundantes, pero, también, va a suponer la creación de otros, como consecuencia de los aumentos de productividad y de las nuevas demandas de los consumidores. A un nuevo perfil de trabajadores, creativos y centrados en desarrollar y determinar la mejor forma de aplicar la inteligencia artificial, se unirá otra clase de empleos relacionados con el mantenimiento, el funcionamiento y la regulación de estas tecnologías emergentes y la creación de Empleos que nunca habrían existido sin la IA (Lund et al., 2019).

\section{Referencias}

Arrieta Rodríguez, D. A., Lora Guzmán, H., \& Sánchez González, J. (2018). Análisis de las empresas de Cartagena del sector logístico portuario basado en el modelo de configuración estratégica de Danny Miller. Aglala, 9(1), 62-90. Recuperado de http://revistas.curnvirtual.edu.co/i ndex.php/aglala/article/view/1182

Barragán Galindo, F., \& Zarete Escobar, S. I. (2017). MODELO RESIGNIFIACIÓN PARA INCENTIVAR EL USO DE LAS TIC EN LA PRÁCTICA PEDAGÓGICA EN COREDUCACIÓN. Revista Innova ITFIP, 1(1), 36-46. Recuperado a partir de http://revistainnovaitfip.com/index .php/innovajournal/article/view/11 Bughin, J., Hazan, E., Lund, S., Dahlström, P., Wiesinger, A., \& Subramaniam, A. (2018). Skill shift: Automation and the future of the workforce. McKinsey Global Institute. Retrieved on, 10 
REVISTA INNOVA ITFIP, 6(1).106-119 JUNIO 2020

Calderón Saque, E. A. (2018). EDUCACIÓN ECONÓMICA Y FINANCIERA, UNA SOLUCIÓN A LA EXCLUSIÓN. Revista Innova ITFIP, 3(1), 44-51. Recuperado a partir de http://revistainnovaitfip.com/index .php/innovajournal/article/view/38

Canto, J. V., \& Mejía, C. B. (2007). Los indicadores financieros y el Valor Económico Agregado (EVA) en la creación de valor. Industrial Data, 10(1), 42-47.

Ceballos, O. I., Mejía Castellanos, L. A., \& Arango Medina, D. (2019). AUDITORIA DE USABILIDAD DE HERRAMIENTAS IMPLEMENTADAS EN PLATAFORMAS VIRTUALES PARA OFERTAR SERVICIOS CON RESPONSABILIDAD SOCIAL. Revista Innova ITFIP, 5(1), 64-77. Recuperado a partir

http://revistainnovaitfip.com/index .php/innovajournal/article/view/56

Fahrni, S., Jansen, C., Michael John, M., Kasah,T., Körber,B \& Mohr, N. (2020). Coronavirus: Industrial IoT in challenging times. McKinsey \& Company

García Guiliany, J., Paz Marcano, A., \& Cantillo Campo, N. (2019). Estrategia y habilidades para la competitividad: caso de pymes del sector construcción en Barranquilla. Aglala, 10(1), 312339. Disponible en http://revistas.curnvirtual.edu.co/i ndex.php/aglala/article/view/1349

Geissbauer, G., Lübben , E., Schrauf, S., Pillsbury,S. (2018) Global Digital Operations Study 2018
Digital Champions. (2018) PwC. All rights reserved

Granados, C., Varghese, R., Baranova, V., Dechalendar, A. \&

Wallenstein, J., (2019) Automation, Skills and the Future of Work: What do Workers Think? IMF Working Paper. IMF Working Paper European Department

Hagel, J.,Seely, B \& Wooll M (2019). Skills change, but capabilities endure. Deloitte Insights

Hoyos Botero, C. (2000). Un modelo para investigación documental: guía teórico-práctica sobre construcción de Estados del Arte con importantes reflexiones sobre la investigación. Medellín: Señal Editora.

Lund, S., Manyika, J., Hilton, L., Dua, A., Hancock, B., Rutherford, S., \& Macon, B.(2019) The future of work in America. McKinsey Global Institute.

Manyika, M., Lund, S \& Chui,M. (2017 ) JOBS LOST, JOBS GAINED: WORKFORCE TRANSITIONS IN A TIME OF AUTOMATION. MGI

Martínez Sahagún, D. (2019). CONSIDERACIONES PARA APLICAR LOS PRINCIPIOS DE LA TEORÍA DEL ACTOR-RED A LA COMUNICACIÓN PÚBLICA DE LA CIENCIA Y LA TECNOLOGÍA. Revista Innova ITFIP, 5(1), 78-88. Recuperado a partir de http://revistainnovaitfip.com/index .php/innovajournal/article/view/74 Marquina, P., Álvarez, G., Guevara, D., \& Guevara, R. (2013). Guía de Trabajos de la Literatura. 
REVISTA INNOVA ITFIP, 6(1).106-119 JUNIO 2020

Moreno, J., \& Rivas, S. (2002). La Administración

Financiera. Editorial Continental. México Mexican Ministry of Economy. (2016). Crafting the future: A roadmap for industry 4.0 in Mexico.

Morrar, R., Arman, H., \& Mousa, S. (2017). The fourth industrial revolution (Industry 4.0): A social innovation

perspective. Technology

Innovation Management

Review, 7(11), 12-20.

Moueddene, K., Coppola M,. Wauters P., Ivanova M., Paquette J. \& Ansaloni (2019). Expected skills needs for the future of work Understanding the expectations of the European workforce. Deloitte Insights

OCDE (2018). Towards the implementation of the G20 roadmap for digitalization: skills, business dynamics and competition. Paris: OECD

Palma Cardoso, E., Leyva Ardila, F., Marroquin, M., \& Torres, N. (2017). Impacto de los egresados del programa de contaduría pública del ITFIP en el mercado laboral. Revista Innova ITFIP, 1(1), 98-103. Recuperado a partir

de

http://www.revistainnovaitfip.com /index.php/innovajournal/article/vi ew/18

Palma Cardoso, E., Caycedo Riaño, M., Guzmán, R.A., Varon Giraldo, O. y Ruiz Conde, S. 2019. Estrategias de mejoramiento a partir de la responsabilidad social y ambiental en los procesos de producción en la agroindustria arrocera del sur oriente del Tolima. Aglala. 10, 2 (dic. 2019), 38-59. DOI:https://doi.org/10.22519/221 57360.1431.

Peña Estrada, C.C.., Velázquez García, L., \& García Mendoza, R. (2017). Tecnologías De Información En El Aprendizaje En La Facultad De Contaduría Y Administración UAQ, MÉXICO. Revista Innova ITFIP, 1(1), 47-57. Recuperado a partir de http://revistainnovaitfip.com/index .php/innovajournal/article/view/12 Ryder, G., Sarah, M (2019) Trabajar para un futuro más prometedor Comisión Mundial sobre el Futuro del Trabajo Oficina Internacional del Trabajo - Ginebra: OI

Varón Giraldo, O., Gutiérrez Delgado, D. M., \& Rodríguez López, W. D. (2018). Procesos contables aplicados por los comerciantes al por menor del municipio de icononzo - tolima para identificar necesidades de capacitación en el área. Revista Innova ITFIP, 3(1), 27-34. Recuperado a partir de http://revistainnovaitfip.com/index .php/innovajournal/article/view/36 Velásquez García, L. A., García Mendoza, R. del C., Rincón García, B., \& Peña Estrada, C. C. (2018). TI EN EL PROCESO DE INGRESO EN UNA IES EN MÉXICO. Revista Innova ITFIP, 3(1), 53-63. Recuperado a partir de http://revistainnovaitfip.com/index .php/innovajournal/article/view/39

Weier, J., Dinesh, M. \& Venkataraju, R. (2019) Skills Change, But Capabilities Endure. Deloitte Insights 
REVISTA INNOVA ITFIP, 6(1).106-119 JUNIO 2020

Weller, P., Dollar, B., Manolian, H., Mock, L. \& Hussain., A. (2019) A The future of work in manufacturing A DELOITTE SERIES ON THE SKILLS GAP AND THE FUTURE OF WORK IN MANUFACTURING

\section{Reportes}

Deloitte. (2018) Reimagining Human Resources.The future of the enterprise demands a new future of HR.

Recuperado https://www2.deloitte.com/content /dam/Deloitte/global/Documents/ HumanCapital/gx-human-capitalthe-future-of-the-enterprise.pdf

Deloitte (2020) Understanding the sector impact of COVID-19. Deloitte Insights. Recuperado https://www2.deloitte.com/global/ en/pages/about-

deloitte/articles/covid-

19/understanding-the-sectorimpact-of-covid-19--engineering--const.html

PwC. (2016). Industry 4.0: Building the digital enterprise. Web, 1-36. https://doi.org/10.1080/01969722. 2015.1007734

PwC. (2017). La inteligencia artificial, impulsara el pib mundial. Web

Recuperado

https://www.pwc.es/es/salaprensa/notas-prensa/2017/lainteligencia-artificial-impulsarapib-mundial.html

PwC (2018). Price waterhouse Coopers. (2018). Tecnología en el trabajo. Web

Recuperado https://www.pwc.com/mx/es/tecno trabajo.html

PwC. $\left(2020^{a}\right)$. CFO Pulse: Impactos financieros del COVID-19 en las empresas. Web

Recuperado https://www.pwc.com/mx/es/gesti on-de-crisis/covid-19/cfo-pulsesurvey.html

PwC. (2020b). 6 de cada 10 cfo apostara por el trabajo remoto permanente una vez se decrete la vuelta a los centros de trabajo. Web Recuperado https://www.pwc.com/mx/es/prens a/2020/cfo-survey-27042020.html 